\title{
Verunreinigungs- und Alterungseffekte in YSZ
}

\author{
Corinna Vonau ${ }^{1,2}$, Rainer Ginzel $^{1}$, Lothar Diehl'; Christian Ohly ${ }^{1}$, Sigrid Meyer ${ }^{1}$, Ulrich Guth ${ }^{2}$ \\ Robert Bosch GmbH, Wernerstraße 51, D-70469 Stuttgart, Germany \\ Technische Universität Dresden, Fachbereich für Physikalische Chemie und Elektrochemie, \\ Bergstraße 66b, D-01062 Dresden, Germany
}

\begin{abstract}
Kurzzusammenfassung
Ein Ziel bei der Entwicklung von sauerstoffsensitiven Hochtemperatursensoren sind schnellere Ansprechzeiten bei niedrigeren Arbeitstemperaturen. Um vergleichbare Kenndaten bei tieferen Temperaturen zu erreichen, sind eine hohe lonenleitfähigkeit des Festelektrolyten und geringe elektrochemische Durchtrittswiderstände notwendig. Der mit der Alterung zunehmende Widerstand des Festelektrolyten wird durch Verunreinigungen beeinflusst [1]. Bekannt ist, dass Siliziumdioxid $\mathrm{SiO}_{2}$ die Dynamik von Platinelektroden/Festelektrolytelektroden (Pt/YSZ, Yttriumdioxid stabilisierten Zirkondioxid), die Anwendung in SOFCs (engl. solid oxide fuel cell, Festoxidbrennstoffzellen) oder Lambdasonden finden, verschlechtert [1-4]. Der Einwirkung von $\mathrm{SiO}_{2}$ steht in Diskussion, die Leistungsfähigkeit, die mechanische Festigkeit und die Alterungsstabilität solcher Elektrodensysteme zu vermindern [5].

Um den Einfluss solcher Verunreinigungen auf YSZ-Substrate zu untersuchen, wurde $\mathrm{SiO}_{2}$ als Verunreinigung in Mengen von 50 ppm bis 10600 ppm zum Substrat gegeben und dieses vor und nach Alterung charakterisiert. Die Ergebnisse elektrochemischer Charakterisierung, rasterelektronenmikroskopischer (engl. scanning electron microscope, SEM) und rastertransmissionselektronenmikroskopischer (engl. scanning transmission electron microscope, STEM) Aufnahmen, sowie Kathodolumineszenzuntersuchungen (engl. cathodic luminescence, CL) liefern eine mögliche $\mathrm{Er}$ klärung für Schädigungen, die durch verstärkte Bildung von monoklinem $\mathrm{ZrO}_{2}$ und sich an den Korngrenzen bildende Glasphasen hervorgerufen werden. Verschiedene Schädigungsmechanismen können daraufhin diskutiert werden.
\end{abstract}

Schlüsselwörter: Platin, stabilisiertes Zirkondioxid YSZ, Sauerstoffsensor, Siliziumdioxid $\mathrm{SiO}_{2}$, Glasphase, hydrothermale Alterung

\section{Einleitung}

Die Suche nach neuen, leistungsfähigeren Materialien für Festelektrolyt-Sauerstoffelektroden, die in SOFCs (engl. solid oxide fuel cell, Festoxidbrennstoffzellen) oder Sauerstoffsensoren verwendet werden, wurde besonders in den letzten 15 Jahren vorangetrieben $[6,7]$. Das am besten untersuchte Sauerstoffsensorsystem ist dabei Platin auf dotierten Zirkondioxiden, wie Pt/YSZ (Yttriumoxid stabilisiertes Zirkondioxid) [8].

Die Oxidionenleitfähigkeit des YSZ ist in der kubischen und tetragonalen Kristallstruktur wesentlich größer als im monoklinen $\mathrm{ZrO}_{2}$. Die kubische Modifikation cYSZ, die ab einer Dotierung von $8 \mathrm{~mol}-\% \mathrm{Y}_{2} \mathrm{O}_{3}$ stabil ist, ist leitfähiger als die teilstabilisierte, tetragonale Form tYSZ (z.B. mit nur 4,5 mol- $\% \mathrm{Y}_{2} \mathrm{O}_{3}$ dotiert). Die Umwandlung in die monokline Form durch hydrothermale Alterung geschieht offensichtlich beim teilstabilisierte $\mathrm{ZrO}_{2}$ (tYSZ) schneller als beim cYSZ $[9,10]$. Dabei sind die ablaufenden Mechanismen und Einfluss nehmenden Parameter wie Zusammensetzung oder äußeren Bedingungen für Alterung und Vergiftung noch wenig untersucht und weitgehend ungeklärt [11].

Besonderen Einfluss auf Funktion und Leistungsfähigkeit von festelektrolytbasierten Elektrodensystemen nehmen Verunreinigungen, wobei auch deren Wirkung im Einzelnen bisher kaum bekannt ist [2-5,12].

Ziel dieser Arbeit ist es, den Einfluss von Siliziumdioxid $\mathrm{SiO}_{2}$ als Verunreinigung im Festelektrolyten systematisch, insbesondere hinsichtlich der Alterung zu untersuchen, um durch ein vertieftes Verständnis der Alterungsphänomene Elektrodensysteme hinsichtlich schnellerer Ansprechzeiten, niedrigerer Arbeits- 
temperaturen sowie verbesserter Alterungsbeständigkeit zu optimieren.

\section{Experimentelles}

Verschiedene tYSZ-Substrate, stabilisiert mit 4,5 mol- $\% \mathrm{Y}_{2} \mathrm{O}_{3}$ mit und ohne $1 \% \mathrm{SiO}_{2}$-Zusatz wurden zum Aufbau einfacher, symmetrischer Pt/YSZ-Elektrodensysteme verwendet (Abb. 1).

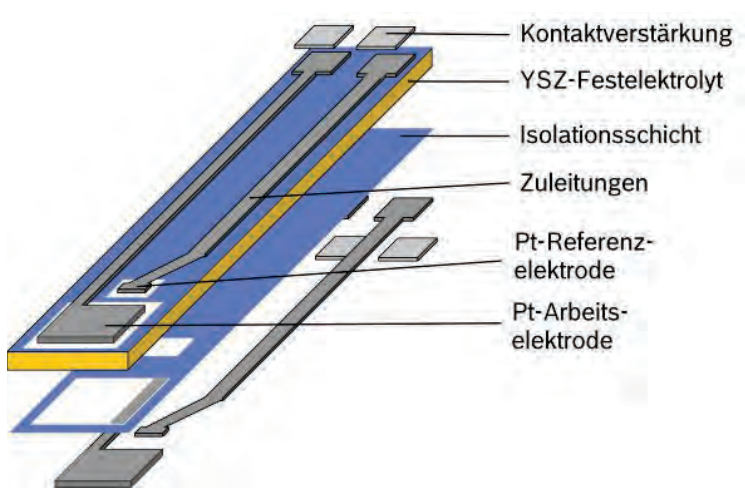

Abb. 1: Schematische Darstellung des genutzten Layouts mit vereinfachtem, symmetrischem Elektrodendesign.

Die Substrate wurden zur hydrothermalen Alterung $6 \mathrm{~h}, 12 \mathrm{~h}$ bzw. $18 \mathrm{~h}$ bei $15 \mathrm{bar}$ und $200{ }^{\circ} \mathrm{C}$ autoklaviert, wodurch der Betriebszustand des Elektrodensystems unter extremen Bedingungen nachgestellt und eine Schädigung beschleunigt werden soll. Die Substrate wurden vor und nach der Alterung mittels Rasterelektronenmikroskop (SEM), Kathodolumineszenz (CL), Rastertransmissionselektronenmikroskop (STEM) und elektrochemischer Impedanzspektroskopie (EIS) charakterisiert.

\section{Ergebnisse}

Monoklines YSZ kann mittels CL nachgewiesen werden, da dieses, im Gegensatz zu seiner tetragonalen oder kubischen Form, durch Auftreffen eines Elektronenstrahls angeregt wird und elektromagnetische Strahlung im sichtbaren Bereich emittiert. Nach Autoklavieren zeigen die Substrate monokline Umwandlungsschichten an den äußeren Rändern des YSZ, wobei die Dicke der Umwandlungsschichten abhängig von der Autoklavierzeit und dem $\mathrm{SiO}_{2}$-Gehalt im YSZ-Substrat ist. Abbildung 2 zeigt eine solche Umwandlungsschicht an einem $18 \mathrm{~h}$ autoklavierten, $\mathrm{SiO}_{2}$-freien Substrat. Die monokline Umwandlungsschicht dringt $15 \mu \mathrm{m}$ in das YSZ-Substrat ein. $\mathrm{SiO}_{2}$-haltige Substrate zeigen 2 - 3 mal größere monokline Umwandlungsschichten als $\mathrm{SiO}_{2}$-freie.

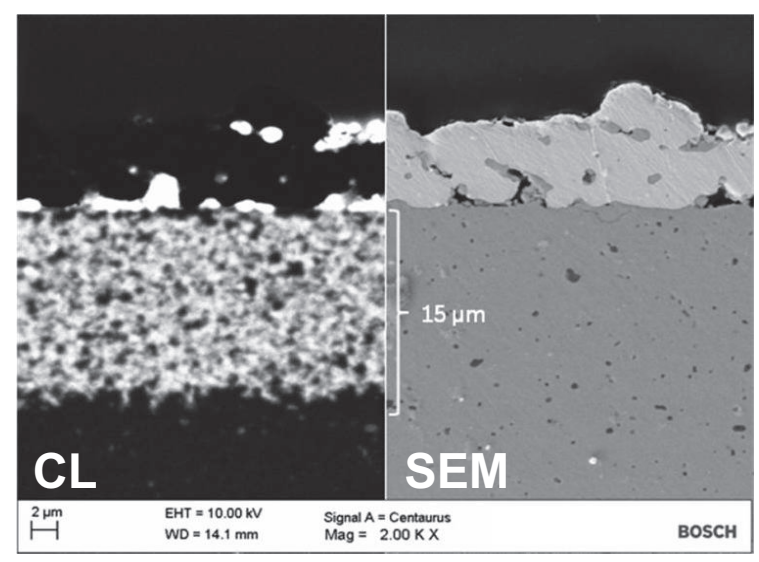

Abb. 2: REM/KL-Aufnahme eines $18 \mathrm{~h}$ autoklavierten $\mathrm{SiO}_{2}$ freien Substrates. Eine $15 \mu \mathrm{m}$ monokline Schicht kann ist durch intensives Leuchten im KLBild sichtbar.

Durch STEM-Aufnahmen lassen sich monoklin umgewandelte YSZ-Körner durch Zwillingsbildung darstellen (Abbildung 3). Bei $\mathrm{SiO}_{2}$-haltigen Substraten zeigen die Aufnahmen außerdem segregierte Phasen entlang der Korngrenzen, in denen sich Si nachweisen lässt.

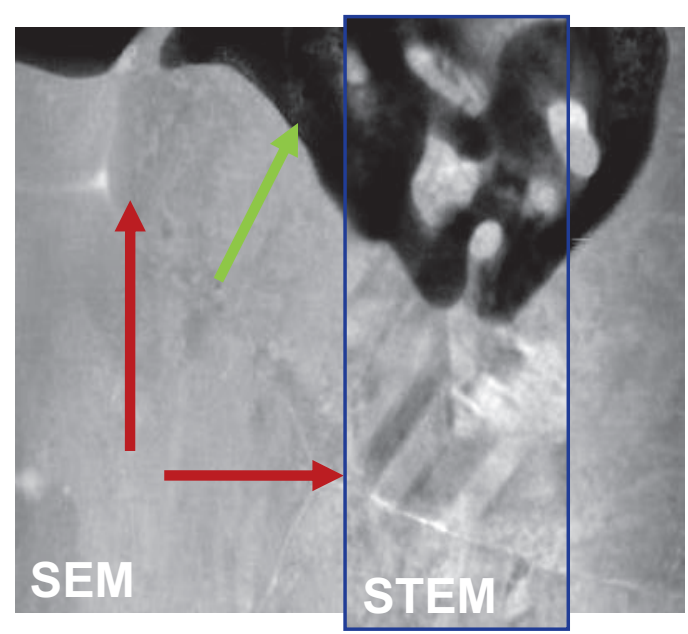

Abb. 3: REM/STEM Aufnahme eines $\mathrm{SiO}_{2}$-haltigen, gealterten Pt/YSZ-Substrates. Eine Si-haltige Phase isoliert YSZ-Körner (rot), wodurch eine monokline Transformation durch Zwillingsbildung von tetragonalen $\mathrm{ZrO}_{2}$-Körnern induziert wird. Am Pt/YSZ-Interface ist kein Si zu finden (grün).

Mittels EIS können Bulk- und Korngrenzwiderstand durch ihre unterschiedlichen frequenzabhängigen Relaxationszeiten bei einer Temperatur $\mathrm{T}=350^{\circ} \mathrm{C}$ voneinander trennen werden. Durch zwei in Reihe angeordnete RCGlieder lassen sie sich einem Ersatzschaltbild zuordnen (Abbildung 4a und 5a). Bei kleineren Frequenzen werden die Relaxationszeiten 
größer, sodass sich außer der Leitfähigkeit im Festkörper auch der Elektrodenprozess separierbar ist. Aus Impedanzmessungen bei verschiedenen Temperaturen lassen sich Aktivierungsenergien $E_{A}$ für die Bulk- und Korn-

a)

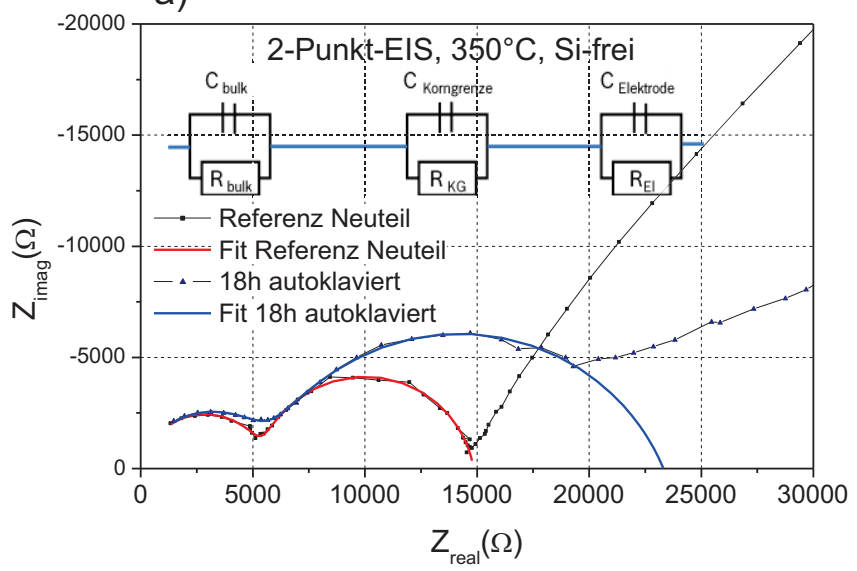

grenzleitfähigkeit abschätzen (Abb. 4b und 5b). Durch hydrothermale Alterung verändern sich diese, wobei momentan noch keine schlüssige Erklärung möglich ist.

Abb. 4: Elektrochemische Charakterisierung $\mathrm{SiO}_{2}$-freier Substrate nach Oh (Referenz, Ref) und 18h Autoklavierzeit.

a) 2-Punkt-Impedanzspektroskopie bei $T=350^{\circ} \mathrm{C}$ zur Trennung von bulk- und Korngrenz-Widerstand $R_{\text {bulk }}$ und $R_{K G}$ des $t Y S Z$.

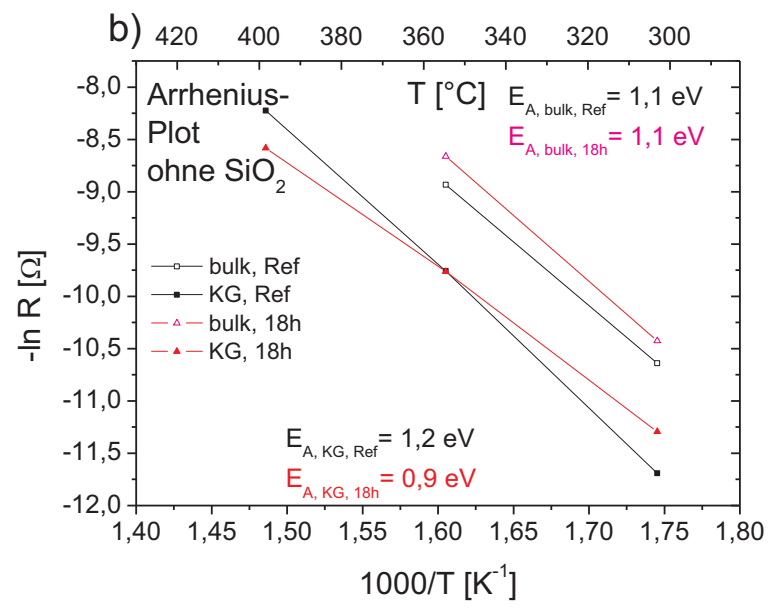

b) Aktivierungsenergien $E_{A}$ für bulk und Korngrenzwiderstand KG bezogen auf Autoklavierzeit.

a)

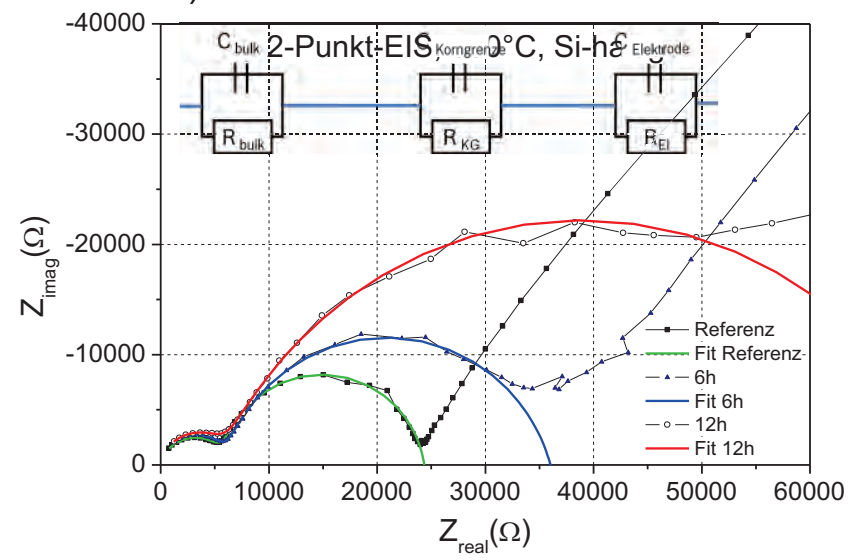

b) $420 \quad 400 \quad 380 \quad 360 \quad 340 \quad 320 \quad 300$

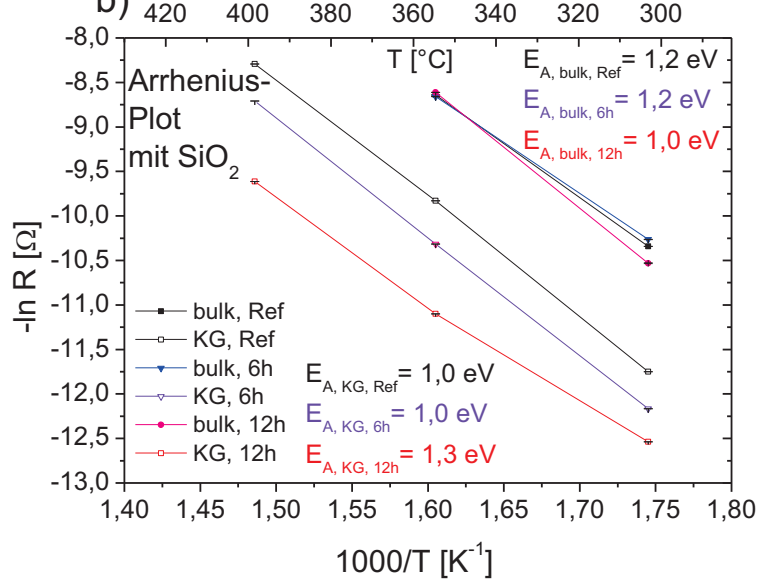

Abb. 5: Elektrochemische Charakterisierung $\mathrm{SiO}_{2}$-haltiger Substrate nach Oh (Referenz, Ref), 6h und 18h Autoklavierzeit.

a) 2-Punkt-Impedanzspektroskopie bei $T=350^{\circ} \mathrm{C}$ zur Trennung von bulk- und Korngrenz-Widerstand $R_{\text {bulk }}$ und $R_{K G}$ des $t Y S Z$. b) Aktivierungsenergien $E_{A}$ für bulk und Korngrenzwiderstand KG bezogen auf Autoklavierzeit. 
Abbildung 6 und Tabelle 1 zeigen die Zunahme des Verhältnisses von neuem und gealtertem Widerstand in Abhängigkeit zur Autoklavierzeit.

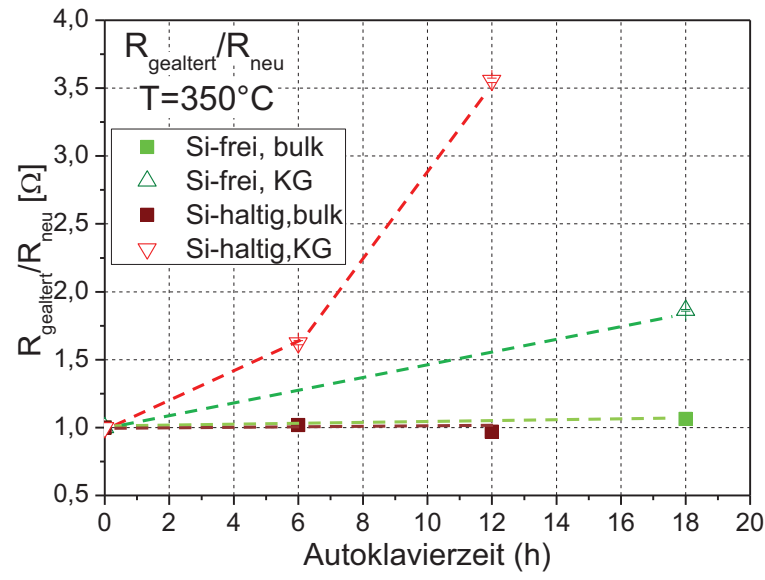

Abb. 6: grafische Darstellung des Verhältnisses gealterter/Referenz Widerstand.

Tab. 1: Verhältnis gealterter/Referenz Widerstand

\begin{tabular}{|c|c|c|}
\hline \multirow{2}{*}{$\begin{array}{c}\mathrm{SiO}_{2^{-}} \\
\text {frei }\end{array}$} & $\mathrm{R}_{\text {bulk,18h }} / \mathrm{R}_{\text {bulk, Ref }}$ & Faktor \\
\cline { 2 - 3 } & $\mathrm{R}_{\mathrm{KG}, 18 \mathrm{~h}} / \mathrm{R}_{\text {KG, Ref }}$ & 2,0 \\
\hline \multirow{3}{*}{$\begin{array}{c}\mathrm{SiO}_{2^{-}} \\
\text {haltig }\end{array}$} & $\mathrm{R}_{\text {bulk,6h }} / \mathrm{R}_{\text {bulk, Ref }}$ & 1,0 \\
\cline { 2 - 3 } & $\mathrm{R}_{\mathrm{KG}, 6 \mathrm{~h}} / \mathrm{R}_{\mathrm{KG}, \text { Ref }}$ & 1,6 \\
\cline { 2 - 3 } & $\mathrm{R}_{\text {bulk,12h }} / \mathrm{R}_{\text {bulk, Ref }}$ & 1,0 \\
\cline { 2 - 3 } & $\mathrm{R}_{\mathrm{KG}, 12 \mathrm{~h}} / \mathrm{R}_{\mathrm{KG}, \text { Ref }}$ & 3,6 \\
\hline
\end{tabular}

Die Zunahme des Korngrenzwiderstandes eines $6 \mathrm{~h}$ autoklavierten, $\mathrm{SiO}_{2}$-haltigen Substrates um einen Faktor 4 zeigt den stärkeren $\mathrm{Al}$ terungseffekt durch die Verunreinigung im Gegensatz zu einem nur um Faktor 2 gestiegenen Korngrenzwiderstand eines $\mathrm{SiO}_{2}$-freien Substrates nach $18 \mathrm{~h}$ hydrothermaler Alterung.

\section{Diskussion}

Die hydrothermale Alterung beschleunigt die monokline Umwandlung der tetragonalen Kristallstruktur des $\mathrm{ZrO}_{2}$ durch einen forcierten Einbau von $\mathrm{OH}^{-}$-Ionen auf freie Leerstellen im Kristallgitter [10,11]. Die monokline Struktur behindert eine Oxidionenleitfähigkeit des Festelektrolyten und damit die elektrochemische Aktivität des gesamten Elektrodensystems.

Es konnte gezeigt werden, dass durch tiefer in den Festelektrolyten eindringende, monokline Umwandlungsschichten und durch $\mathrm{SiO}_{2}$ Verunreinigung die hydrothermale Beständigkeit des Festelektrolyten vermindert wird.
Ursache für diese stärkere monokline Umwandlung des Kristallgitters sind die in $\mathrm{Ab}$ bildung 2 gezeigten Si-haltigen Phasen, die sich entlang der Korngrenzen segregieren.

$\mathrm{SiO}_{2}$ als Verunreinigung ist in der Lage eine Natrium- und Aluminium-haltige Glasphase auszubilden [2-4,13]. In solchen Glasphasen kann sich außerdem Yttrium lösen, das den YSZ-Körnern entzogen wird und damit die Phasenstabilität der tetragonalen oder kubische Kristallstruktur herabsetzt und zusätzlich eine monoklinen Transformation begünstigt [14].

Der Bulkanteil des Festelektrolytwiderstands im tYSZ wird weder durch die Zugabe von $\mathrm{SiO}_{2}$ noch durch hydrothermale Alterung beeinflusst. Anders ist dagegen der Einfluss auf den Korngrenzanteil. Durch Zugabe von $\mathrm{SiO}_{2}$ und damit verbundenen segregierenden Glasphasen entlang der Korngrenzen wird die Korngrenzleitfähigkeit verkleinert, was durch die höheren Korngrenzwiderstände in den Referenzimpedanzen ersichtlich wird (Abbildung 4a und 5a). Nach hydrothermaler Alterung wird der Unterschied im Korngrenzwiderstand von $\mathrm{SiO}_{2}$-haltigen $\mathrm{zu} \mathrm{SiO}$-freien noch deutlicher. Auch damit wird der Einfluss destabilisierender Si-haltiger Glasphasen bestätigt.

\section{Schlussfolgerung}

$\mathrm{SiO}_{2}$ als Verunreinigung in Pt/YSZ- Elektroden führt zu einem größeren Festelektrolytwiderstand und durch hydrothermale Alterung zu einer stärkeren monoklinen Umwandlung des Festelektrolytkristallgitters als bei $\mathrm{SiO}_{2}$-freien Substraten. Die Zugabe von $\mathrm{SiO}_{2}$ hat keinen Einfluss auf den Bullkwiderstand der YSZ-Körner, wie anhand von elektrochemischen Impedanzspektren des Festelektrolyten, sowie deren unveränderten Aktivierungsenergien gezeigt werden konnte.

Durch $\mathrm{SiO}_{2}$ im Festelektrolyten wird der Korngrenzwiderstand des Substrates erhöht, was durch isolierende Glasphasen, die sich entlang der Korngrenzen segregieren und die einzelnen YSZ-Körner voneinander isolieren, begründet werden kann. $\mathrm{SiO}_{2}$ führt einerseits $\mathrm{zu}$ einer stärkeren monoklinen Umwandlung des $\mathrm{ZrO}_{2}-$ Kristallgitters während hydrothermaler Alterung, erniedrigt andererseits aber auch die Aktivierungsenergie der Korngrenzleitfähigkeit. Das Eindringen von $\mathrm{OH}^{-}$in den Festelektrolyten kann eine Reaktion mit $\mathrm{SiO}_{2}$ bewirken, die zum Herauslösen und Abtransportieren von $\mathrm{SiO}_{2}$ aus dem Elektrodensystem beitragen kann. Dadurch werden die Korngrenzen aufgelockert und Platz für eine monokline Transformation des $\mathrm{ZrO}_{2}$-Kristallgitters ermöglicht, dessen Vo- 
lumenzunahme $3 \%$ beträgt. Auch durch die hydrothermale Alterung wird die Aktivierungsenergie der Korngrenzleitfähigkeit abgesenkt, allerdings gibt es dafür momentan keine schlüssige Begründung.

Zusammenfassend senkt $\mathrm{SiO}_{2}$ als Verunreinigung in Elektrodensystemen nicht nur die lonenleitfähigkeit des Festelektrolyten, sondern führt auch zu einer verstärkten Alterung unter hydrothermalen Bedingungen.

Ungeklärt ist bisher der Einfluss von $\mathrm{SiO}_{2}$ auf die Elektrodenprozesse.

\section{Referenzen}

[1] M. Mogensen, Solid State lonics, 123-129 150 (2002), 10.1016/S0167-2738(02)002692

[2] A. Hughes, S. Badwal, Solid State lonics 46, 265-274 (1991), 10.1016/01672738(91)90225-Z

[3] S.P.S. Badwal, J. Drennan, International Workshop on Interfaces of Ceramic Materials, Science of Ceramic Interfaces II, Elsevier, Amsterdam ; New York, p. 71 (1994), 0444816666

[4] E. Mutoro, B. Luerßen, S. Günther, J. Janek, Solid State Ionics 180 1019-1033 (2009), 10.1016/j.ssi.2009.04.012

[5] M. Lankin, Y. Du, C. Finnerty, Journal of Fuel Cell Science and Technology 8, 054001-1 - 054001-7 (2011), $10.1115 / 1.4003980$

[6] S.C. Singhal in: J.T.S. Irvine, P. Connor (Eds.), Solid Oxide Fuels Cells Facts and Figures, Springer London, London, 1-23 (2013), 978-1-4471-4455-7, 978-1-44714456-4

[7] S.J. Skinner, S. Cook, J.A. Kilner, in: J.T.S Irvine, P. Connor (Eds.), Solid Oxide Fuels Cells Facts and Figures, Springer London, London, 181-201 (2013), 978-1-4471-44557, 978-1-4471-4456-4

[8] S.B. Adler, Chem. Rev. 104, 4791-4844 (2004), 10.1021/cr020724o

[9] X. Guo, Chem. Mater. 16, 3988-3994 (2004), 10.1021/cm040167h

[10] J. Chevalier, L. Gremillard, A.V. Virkar, D.R. Clarke, J. Am. Ceram. Soc. 92, 1901-1920 (2009), 10.1111/j.1551-2916.2009.03278.x

[11] X. Guo, J. Phys. Chem. Solids 60, 539-546 (1999), 10.1016/S0022-3697(98)00301-1

[12] J.W. Fergus, J. Power Sources 162, 30-40 (2006), 10.1016/j.jpowsour.2006.06.062

[13] R. Knibbe, G.J. Auchterlonie, T. Mori, A. Lashtabeg, J. Drennan, J. Am. Ceram. Soc.
1494-1500 (2010), 10.1111/j.15512916.2010.03612.x

[14] X. Guo, J. Maier, J. Electrochem. Soc. 148, E121-E126 (2001), 10.1149/1.1348267 